\title{
New dimension for Mendeleev
}

After more than a century, what can there be to say about the Periodic Table that is new? Merely to ask a question of such disarming simplicity, of course, signals that somebody, indeed, has something new to say.

EVERYBODY remembers the first occasion, usually in a school classroom, when Mendeleev's Periodic Table first presented itself as one of the great organizing principles of nature. The idea that all 92 natural elements might be fitted into a simple diagram on a sheet of paper must for many people have seemed the quickest way of holding the world's secrets in the head.

To be told, usually later, that the good sense of Mendeleev's conjecture about the similarities between the different elements (first made in the year Nature was founded) had been confirmed by everything since learned from quantum theory could only have served to confirm Mendeleev's reputation as one of almost magical insight. And it is, after all, not just a legend, but the truth, that he predicted that there would be found the element now called scandium.

Bohr was one of the first to make it clear that, by the 1920 s, even his version of the quantum theory accounted for the regularities, and made plausible some of the irregularities, of the Periodic Table. On the onion model of atomic structure, in each successive completed shell of electrons there are $2 n^{2}$ electrons, where $n$ is an integer greater than or equal to unity and representing the order of occurrence of the electron shell in the onion model. Even young people can be entranced to know that the factor " 2 " arises simply because there are two allowed directions of electron spin.

But which version of the table should be kept in mind? Classroom walls are hung with two - a more or less square array of symbols (with hydrogen and helium displayed separately at the top) and another shaped like a schematic drawing of an Aztec or Babylonian temple, consisting of several superimposed layers with the broadest at the bottom. The two versions differ simply in their arrangements to accommodate elements such as the rare earths, but the result must be to leave many with the impression that Mendeleev had not made up his mind about something of importance.

Luckily, as we all now know, there is no hidden scandal. In adding electrons successively to an empty shell in an onion model of an atom, the first eight electrons go where they are expected to fit, in the $s$ and $p$ states (holding two and six respectively), but then it proves to be less energetically favourable to accommodate succeeding electrons in the same shell, into what should be the $d$ state, with a capacity for ten. At least two of them slot more easily into the next higher shell, whereupon the unfilled states in the next innermost shell can be filled (which process accounts for the transition elements from scandium to nickel inclusive).

The implication is that the eight-fold periodicity in the Periodic Table (and about the only true periodicity there is in it), represented by the elements from lithium to neon inclusive, and from neon to argon, is strictly spurious in origin. With elements whose atomic number is greater, there are inevitably more complicated transitions, as with lanthanum, the first of the rare earths, where, with the $6 s$ state as well as the $5 s$ and $5 p$ states filled, the electron added to the full complement of barium's electrons goes into the empty $5 d$ state rather than into the still empty $4 f$ state.

It is of some interest that this curious behaviour, and the two series of transition elements as well as the rare earths and the actinides (mostly artificial elements), owe their existence to the present value of the electron charge. If it were very different, even Mendeleev might not have been able to spot regularities among the data with which he was confronted.

Many of the irregularities are more particular and more perplexing. Everybody can accept that elements such as helium and argon have much in common, even if the collective term "inert gases" has recently been rendered a misnomer, but what does carbon have in common with tin and lead, all of them members of what are called the Group 1V elements in the table? More generally, why in any group of elements with supposed affinity are those with greater atomic number more inclined to form cations as if they were metals?

These are some of the questions now taken up by Leland C. Allen of Princeton University (J.Amer. Chem. Soc.114, 1,310; 1992) and answered with the simple declaration that it is "clear that something is missing". The Periodic Table, he says, cannot be complete as a two-dimensional array, whatever its shape. He says there must be a missing dimension, which he argues must somehow be a function of the electronic energy of the atoms concerned. $\mathrm{He}$ calls the missing dimension "configuration energy" and proceeds to define it, at least formally, as the average one-electron valence shell energy of a ground-state free atom.

Nobody will blame Mendeleev for not having thought of that. Moreover, con- figuration energy as defined can in principle be measured by spectroscopists. It is only necessary to measure the ionization potentials of the valence electrons (which may differ in the $s, p$ and $d$ states) and then to take an average weighted by the number of the electrons in the different states. But, like all empirically conceived measures - Allen refers to Pauling electronegativity index, dating from 1932, as another - what matters is whether configuration energy resolves enough of the inconsistencies in the Periodic Table to win the hearts and minds of men with an interest in these matters.

What Allen is most pleased with is that configuration energy as defined rationalises what he calls the metalloid band in the table, which is the diagonal line in the rectangular representation of the table reaching from boron (element 5) to antimony (element 51). All the elements to the left are metals and have small configuration energy, all those to the right are non-metals with higher configuration energy - and those at the boundary have virtually the same configuration energy as each other.

Allen argues that his index, which he says subsumes Pauling's, "uniquely qualifies" as the extra dimension missing from Mendeleev's table. On the evidence presented, that may well be the case, although it will be interesting to see whether it can make predictions about, say, the properties of simple compounds that have not yet been made which are arresting enough to compel general attention.

A more obvious difficulty is that the extra dimension may be half a century too late. At the beginning, the Periodic Table was most spectacularly useful as a predictive tool, but by 1932 it had become mostly a mnemonic. Since then, two generations have learned to rationalise some of the irregularities of the Periodic Table by armwaving of a pseudo-theoretical character. That the inert gases are more or less chemically inert is justified by the extra binding energy of electrons in a complete valence shell of electrons whose orbits are symmetrically hybridized with each other, which means that excitation to the next higher energy level (as required in some virtual sense, at least, to form a chemical bond) is relatively difficult. Those who use such arguments do not, of course, claim to have made the calculations, but that is no greater shame now than some of the other arguments of the same kind used in the past century.

John Maddox 